\title{
Cooling compact stars and phase transitions in dense QCD
}

\author{
Armen Sedrakian \\ Institute for Theoretical Physics, J. W. Goethe University, \\ Max-von-Laue-Str. 1, D-60438 Frankfurt am Main, Germany
}

Received: date / Revised version: date

\begin{abstract}
We report new simulations of cooling of compact stars containing quark cores and updated fits to the Cas A fast cooling data. Our model is built on the assumption that the transient behaviour of the star in Cas A is due to a phase transition within the dense QCD matter in the core of the star. Specifically, the fast cooling is attributed to an enhancement in the neutrino emission triggered by a transition from a fully gapped, two-flavor, red-green colorsuperconducting quark condensate to a superconducting crystalline or an alternative gapless, color-superconducting phase. The blue colored condensate is modeled as a Bardeen-Cooper-Schrieffer (BCS)-type color superconductor with spin-one pairing order parameter. We study the sensitivity of the fits to the phase transition temperature, the pairing gap of blue quarks and the time-scale characterizing the phase transition (the latter modelled in terms of a width parameter). Relative variations in these parameter around their best fit values larger than $10^{-3}$ spoil the fit to the data. We confirm the previous finding that the cooling curves show significant variations as a function of compact star mass, which allows one to account for dispersion in the data on the surface temperatures of thermally emitting neutron stars.
\end{abstract}

PACS. 97.60.Jd Neutron stars - 26.60.-c Nuclear matter aspects of neutron stars - 95.30.Cq Elementary particle processes -74.25 .Dw Superconductivity phase diagrams

\section{Introduction}

The central densities of compact stars (hereafter CSs) can be by a factor of few up to ten times larger than the saturation density of nuclear matter. In this range of densities the mean interparticle distance may become of the order of characteristic size of a baryon, therefore neutrons, protons, and heavier baryons will eventually lose their identity, as their wave-functions start to overlap. Although the detailes of the mechanism of deconfinement are not well understood so far, it is plain that at sufficiently large density the baryonic matter will undergo a deconfinement phase transition to quark matter. Not only the transition itself, but also the phase structure of quark matter is difficult to access, because the densities in CSs will not be large enough for the perturbative QCD to be valid. Astrophysics of CSs offers an avenue to explore and constrain the possible deconfinement phase transition and the properties of dense QCD matter via modeling of CSs containing quark cores [1, 2].

The focus of this work is the effects of the deconfinement phase transition and the phase structure of dense QCD on the cooling of CSs. This discussion extends the previous work on the cooling of hybrid stars containing superconducting quark matter, including the interpretation of the rapid cooling of the CS in Cassiopea A as a phase transition within the QCD phase diagram [3, 4]. Specifically, we report the results of new simulations of cooling of CSs and fits to the Cas A updated data [5], which covers the 10 year period from 2003 to 2013. These data indicates an unprecedented fast cooling of the neutron star

Send offprint requests to: sedrakian@th.physik.uni-frankfurt.de in Cas A, which requires fast transient modeling of this object (as opposed to the familiar long-time-scale modeling of neutron stars, where characteristic timescales of variations are $t \geq 100 \mathrm{y}$ ). The best estimate of Ref. [5] indicates a decline in the temperature of the star $2.9 \pm 0.9 \%$ over 10 years of observation, which requires extremely fast transient cooling of the star over this period. The currently available data cannot be interpreted unambiguously; one reason is the bright and varying supernova remnant background, which makes a definitive interpretation difficult [5]. Another uncertainty arises from adopted constraints on the temperature fitting parameters and the uncertainties of the effective area calibration; for example, the apparent decline in the temperature can be compensated if one allows for variations in the emitting region size [6]. In the following we leave aside the possible uncertainties in the interpretation of the data and focus on its theoretical modeling.

A variety of models account theoretically for the Cas A data. Each class of models has its own specific trigger for onset of rapid cooling around the age of the Cas $\mathrm{A}(t \simeq 300$ y). A class of nucleonic models (i.e., models of CSs containing neutrons, protons, and electrons) attribute the rapid cooling to the onset of Cooper pair-breaking process in CS's superfluid component [7, [8, 9, 10, 11, 12]. The rapid cooling occurs once the temperature drops below the critical temperature $T_{c, n} \simeq 10^{9} \mathrm{~K}$ of phase transition of nucleons to the superluid state. This allows for additional neutrino emission via Cooper-pair breaking and formation (PBF) processes from $S$ wave [13, 17, 18, 19, 20] and $P$-wave condensates [21, 22]. These minimalistic models do not require any additional new 
physics beyond the standard scenario which is supplemented by pair-breaking processes [23].

The Cas A data was also explained in Ref. [24, 25] within a model where modified Urca and bremsstrahlung processes are enhanced compared to rates used in the minimalistic models quoted above by several orders of magnitude due to a softening of pionic modes, as initially discussed in Ref. [26]. Rotational changes in star's composition may induced Urca process as the star slows down, which in turn can cause a Cas A type fast transient [27], which however would require very fine tuning of parameters, as already discussed in Ref. [3].

Color-superconductivity was included in the simulations of CS to account for Cas A data in Refs. [3, 28]. In Ref. [28] the phase transition from purely nucleonic to purely quark matter occurs via mixed phases, whereas Ref. [3] assumes sharp interface between the two; which scenario is realized depends on the poorly known surface energy between the nucleonic and quark phases. We explore here the second possibility.

This paper is organized as follows. In sect.2 we discuss the relevant features of the phase diagram of dense and cold QCD. Section 3 describes the physical input needed for cooling simulations, including neutrino emissivities of various phases and specific heats. The results of numerical simulations are presented in sect. 4 together with the fits to the Cas A data. A summary and conclusions are given in sect.5

\section{The phase structure of dense QCD and massive compact stars}

This section provides the background information on the QCD phase diagram, which is necessary for the understanding of the model of cooling of CSs presented in the following sections. Readers interested in the astrophysical aspects of the modeling can proceed to sect. 3, those interested in the results can go directly to sect. 4

We consider a class of hybrid CSs, which contain dense quark cores surrounded by a nucleonic envelope, with a sharp phase-transition interface between these phases. Stars with such hybrid structure naturally correspond to the most massive members of the sequence of stellar equilibria modelled with an equation of state which contains a phase transition from nucleonic to quark matter. These massive members must be heavy enough to account for the recently observed two-solar mass pulsars PSR J1614-2230 and PSR J0348+0432 [29, 30].

If quarks in the deconfined phase of the star are unpaired (i.e. do not form Cooper pairs) they will cool the star by neutrino emission rapidly to temperatures well below those observed in Cas A [4, 31, 32, 33, 34]. However, cold quark matter is expected to be in one of the conjectured superconducting phases due to the attractive component in the gluon exchange quark-quark interaction [35]. The $\beta$-equilibrium and strange quark mass shift the Fermi surfaces of up and down quarks apart. As a consequence some non-BCS phases can now emerge. Such non-BCS phases include the gapless two-flavor phases [36, 37] or, for example, the crystalline color-superconducting phase [38, 39]. The crystalline-color-superconductivity itself has a multitude of realizations, which differ by the way the translational symmetry is broken by the condensate of

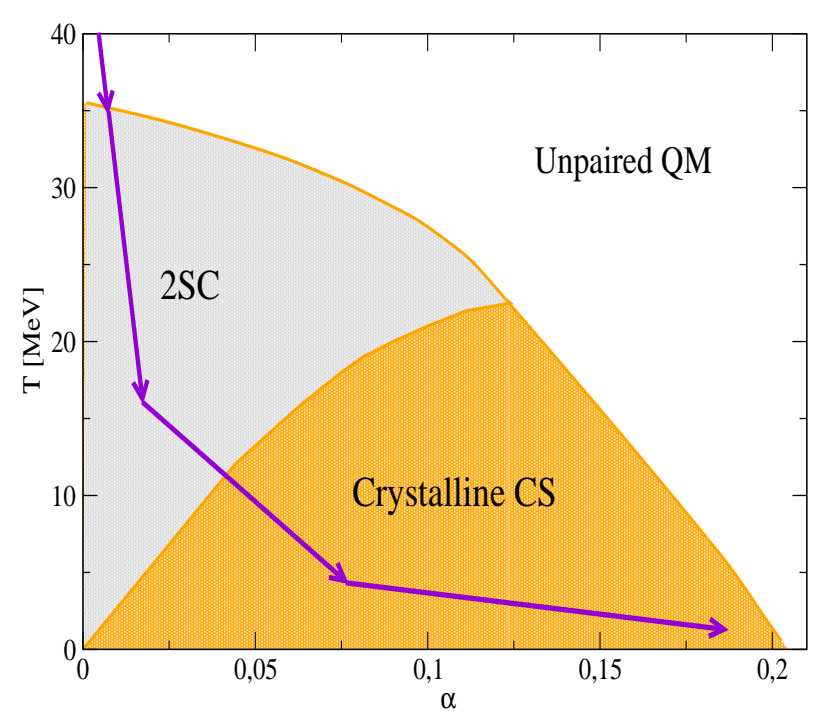

Fig. 1. Phase diagram of color-superconducting quark matter in the temperature $(T)$ and isospin imbalance $\alpha=\left(n_{d}-n_{u}\right) /\left(n_{u}+n_{d}\right)$ plane, where $n_{d}$ and $n_{u}$ are the number densities of $d$ and $u$ flavors of quarks. The arrows schematically show the path in the phase diagram during the cooling of a star.

Cooper pairs carrying finite momentum. For concrete calculations we will assume below the so-called Fulde-Ferrell phase (hereafter FF phase), which is simple to model, but is general enough to preserve a key feature of the crystalline phases, which is the existence of gapless modes on the Fermi surfaces of up and down quarks [38, 39, 40, 41].

Our conjecture concerning the behaviour observed in Cas $\mathrm{A}$ is based on a robust feature of the phase diagram of the two-flavor color superconducting matter, (and, in fact, any twocomponent fermionic systems with attractive interaction). Just below the critical temperature and at not too high isospin asymmetries, measured by parameter $\alpha=\left(n_{d}-n_{u}\right) /\left(n_{u}+n_{d}\right)$, where $n_{d}$ and $n_{u}$ are the number densities of $d$ and $u$ flavors of quarks, the rotational/translational symmetries are unbroken, i.e., the fully gapped 2SC phase is favored (see Fig. 1). At lower temperatures a phase transition to a less symmetrical phase (such as the FF phase) becomes favorable at larger asymmetries, but not large enough to destroy the superconductivity completely. (This would correspond in Fig. 1 to $\alpha>0.2$ ). Note that the phase diagram shown in Fig 1 is valid only in the weak coupling limit, which is the case in the bulk of CSs . (If the coupling is large enough the ensemble makes a transition from 2SC to Bose-Einstein condensed phase without entering crystalline phase; see, for example, Refs. [42, 43, 44] for a generic and most complete phase diagram of a fermionic system with imbalance).

We next explore the consequences of the transition from the symmetrical two-flavor BCS phase to the crystalline phase (in our case the FF phase) for the cooling of CSs and the CS in Cas A. We anticipate such a transition in CSs because a newly born proto-CSs have temperatures of the order of several tens of $\mathrm{MeV}$ and matter in nearly isospin symmetrical $(\alpha \simeq 0)$ state. Within short period of time (minutes to hours) the CS cools to temperatures of the order of $1 \mathrm{MeV}$ and becomes strongly 
isospin asymmetrical as $\beta$-equilibrium among quarks and electrons is established. The arrangement of phases shown in fig. 1 . in the case of $\beta$-equilibrated matter is shown in fig. 2, which is adapted from Ref. [41]. A shell of quark matter phase in the star

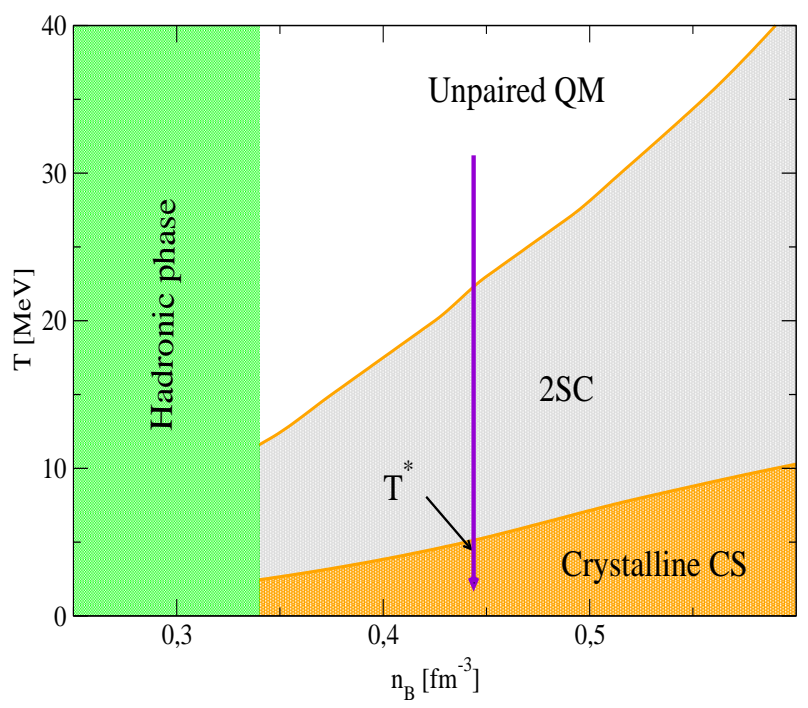

Fig. 2. Phase diagram of the same phases as in fig. 1 but in the temperature and baryon density $\left(n_{B}\right)$ plane for quark matter in $\beta$ equilibrium with electrons. The hadronic phase is shown schematically on the left.

with fixed density will traverse the $T$ - $\alpha$ plane from upper left corner to the lower right corner as shown by arrows in fig. 1 The same path is shown in fig. 2 in the temperature and density plane.

The transition temperature from the $2 \mathrm{SC}$ to the FF phase is denoted by $T^{*}$ and will be treated below as a free parameter. Our fits to Cas A data below will demonstrate that the values obtained from theoretical models are larger than those obtained from fits. This uncertainty is acceptable as the magnitude of the pairing interaction among quarks in the non-perturbative regime of QCD is model dependent and generally not wellknow.

Finally, assuming that the cross-flavor pairing occurs between the green and red colored quarks we need to address the pairing among the remaining blue colored quarks. The strength and the flavor content of pairing among blue quarks is model dependent [45, 46, 47]. Obviously blue-up and blue-down pairing is suppressed by a large mismatch between the Fermi surfaces of the up and down quarks. Pairing is more likely in the color $\mathbf{6}_{S}$ and flavor $\mathbf{3}_{S}$ channel, which is same-flavor and samecolor pairing, therefore it is not affected by the flavor asymmetry [45]. Calculations show that the gaps are in the range 10-100 $\mathrm{keV}$. Again because of uncertainties envolved in the interaction generating the BCS state of blue quarks we treat the blue quark gap $\Delta_{b}$ as a parameter of our model.
Table 1. The table lists the central density $\rho_{c, 14}$, quark core radius $R_{Q}$, quark plus hadronic core radius $R_{Q+H}$, the isothermal core radius $R_{c r}$, the star radius $R$, and the masses of the quark core $M_{Q}$, the hadronic core $M_{H}$, the crust $M_{c r}$, and the total mass $M$. The density is in units of $10^{14} \mathrm{~g} \mathrm{~cm}^{-3}$, the radii in kilometers, and the masses in units of $M_{\odot}$ [4].

\begin{tabular}{ccccccccc}
\hline \hline & & & & & & & \\
$\rho_{c, 14}$ & $R_{Q}$ & $R_{Q+H}$ & $R_{c r}$ & $R$ & $M_{Q}$ & $M_{H}$ & $M_{c r}$ & $M$ \\
\hline \hline & & & & & & & & \\
\hline 5.1 & - & 11.47 & 13.39 & 13.53 & - & 1.03 & 0.07 & 1.10 \\
8.2 & - & 12.57 & 13.55 & 13.57 & - & 1.81 & 0.04 & 1.85 \\
& & & & & & & & \\
\hline & & & & & & & & \\
10.8 & 0.68 & 12.54 & 13.49 & 13.5 & 0.001 & 1.866 & 0.0397 & 1.906 \\
11.8 & 3.41 & 12.40 & 13.31 & 13.32 & 0.093 & 1.802 & 0.0374 & 1.932 \\
21.0 & 6.77 & 11.34 & 11.91 & 11.92 & 0.888 & 1.143 & 0.0191 & 2.050 \\
& & & & & & & & \\
\hline \hline
\end{tabular}

\section{Physical input into simulations}

\subsection{Equilibrium structure of hybrid stars}

Prior to computing the time evolution of CS models with color superconducting phases discussed in the previous section we need to construct the equilibrium models of such stars. Two classes of models were developed and used by us in the studies of color superconducting quark matter. Both classes are based on equations of state (hereafter EoS) with a sharp phase transition between the nuclear core and quark phases; the first class features crystalline color superconducting quark matter in the quark core and nuclear envelope, which is described by a relativistic density functional model with a stiff parameterization of the EoS [48, 49]. The second class of models contains 2SC phase discussed in the previous section and the color flavor locked (CFL) phase at high densities [50, 51]. Both models produce stable sequences of color superconducting CSs with maximal masses above the lower observational bound mentioned above. We model the high-density matter EoS within the effective Nambu-Jona- Lasinio four-fermion interaction model supplemented by pairing interaction between quarks. The nuclear and quark equations of state were matched by a Maxwell construction. If one uses alternative constructions mixed phases will appear, which we do not discuss here (see Refs. [28, 52]). We will assume that strange quarks because of their large mass are not important at the densities relevant to our models. The nucleonic phase itself can contain non-zero strangeness in form of hyperons. But the compatibility of hyperons with large masses of pulsars needs special attention. Indeed their appearance softens the EoS and the maximal masses of neutron stars are lower than those observed. This "hyperonization puzzle" is a fundamental problem on its own right and will not be discussed here (see Refs. [53, 54, 55, 56, 57, 58, 59] for recent discussions of this problem). Below we will exclude the hyperons from the consideration.

The parameters of our models are listed in table 1 . The models with low central densities $\left(\rho_{c} \leq 3 \rho_{0}\right)$ have masses $M \leq$ 
$1.85 M_{\odot}$ and are purely hadronic. The models with larger central densities contain quark cores, with the size of the core increasing with the central density.

\subsection{Neutrino emissivities in quark phases}

We consider first unpaired quark matter. Because quarks are ultra-relativistic the Urca process works without any kinematical restrictions (as opposed to baryonic matter). For two flavor quark matter consisting of $u$ and $d$ flavors of quarks the main modes of neutrino emission are given by

$$
d \rightarrow u+e+\bar{v}, \quad u+e \rightarrow d+v
$$

where $e$ stands for electron, $v$ and $\bar{v}$ electron neutrino and antineutrino. The corresponding emissivity (energy output per unit volume and time) was calculated to leading order in the strong coupling constant $\alpha_{S}$; the emissivity per quark color is given by [60],

$$
\epsilon_{\beta}=\frac{914}{945} G^{2} \cos ^{2} \theta p_{d} p_{u} p_{e} \alpha_{s} T^{6},
$$

where $G$ is the weak coupling constant, $\theta$ the Cabibbo angle, and $p_{d}, p_{u}$, and $p_{e}$ are the Fermi momenta of down quarks, up quarks, and electrons.

The quark pairing modifies the temperature dependence of process (2). If the quark condensate was a BCS-type superconductor, the emission would have been suppressed linearly for $T \simeq T_{c}$ and exponentially for $T \ll T_{c}$, where $T_{c}$ is the critical temperature. However, in the gapless superconductors there is an additional new scale $\delta \mu=\left(\mu_{d}-\mu_{u}\right) / 2$, where $\mu_{u, d}$ are the chemical potentials of light quarks. Depending on the relative ratio of the two scales in the problem, namely $\delta \mu$ and $\Delta_{0}$ defined as the pairing gap for $\delta \mu=0$ the suppression of emissivity is qualitatively different. In terms of the parameter $\zeta=\Delta_{0} / \delta \mu$ one finds that in the $\zeta>1$ regime the Fermi surfaces of quarks are gapped and the emissivity is suppressed as in the case of BCS superconductors [61]. In the opposite case $\zeta<1$ the Fermi surfaces have nodes and particles can be excited around these nodes without energy cost needed to overcome the gap. In the case of the FF phase the shift in the chemical potential is replaced by a more general function - the anti-symmetric in the flavor part of the single particle spectrum of up and down quarks. As a result the parameter $\delta \mu$ is replaced by $\left[\epsilon_{d}(\boldsymbol{Q})-\epsilon_{u}(\boldsymbol{Q})\right] / 2$, where $\boldsymbol{Q}$ is the total momentum of a Cooper pair and $\epsilon_{u / d}$ are the spectra of the $u$ and $d$ quarks.

It is convenient to use below the generic parameterization of the suppression factor of the quark Urca process given by [61]

$$
\epsilon_{\beta}^{r g}\left(\zeta ; T \leq T_{c}\right)=2 f(\zeta) \epsilon_{\beta}, \quad f(\zeta)=\frac{1}{\exp \left[(\zeta-1) \frac{\delta \mu}{T}-1\right]+1} .
$$

A time-independent constant $\zeta$ excludes the possibility of the phase transition between the above mentioned phases. Therefore, following Ref. [3], we adopt temperature dependent (and, therefore, time-dependent) parameter $\zeta(T)$. We use the following parameterization

$$
\zeta(T)=\zeta_{i}-\Delta \zeta g(T),
$$

where $\zeta_{i}$ is the initial (pre-transient in the Cas A case) value, $\Delta \zeta$ the constant change in this function, and the function $g(T)$ describes the transition from the initial value $\zeta_{i}$ to the asymptotic final value $\zeta_{f}=\zeta_{i}-\Delta \zeta$. The transition is conveniently modeled by the following function

$$
g(T)=\frac{1}{\exp \left(\frac{T-T^{*}}{w}\right)+1},
$$

which allows us to control the temperature of transition, controlled by $T^{*}$, and the smoothness of the transition, controlled by the width $w$.

For the blue quarks, which are not involved in the FF pairing, we assume that these are paired as in the BCS phase, as already discussed in the previous section. In that case their emissivity in the superconducting phase is very approximately related to the emissivity in the normal phase

$$
\epsilon_{\beta}^{b}\left(T \leq T_{c}\right) \simeq \epsilon_{\beta}^{b}\left(T>T_{c}\right) J_{b}\left(\frac{\Delta_{b}}{T}\right) \exp \left(-\frac{n \Delta_{b}}{T}\right)
$$

where the function $J_{b}$ is some power-law function and $n$ is an integer of order of unity. We assume $J_{b}=1$ as it is of order unity when $T \sim \Delta_{b}$ and is dominated by the exponential factor for $T \ll \Delta_{b}$. The pairing gap $\Delta_{b}$ will be treated as a fit parameter below.

\subsection{Neutrino emissivities in hadronic phases}

Our treatment of the hadronic phases follows the standard picture of slow cooling, which is supplemented by the pairingbreaking processes in the superfluid phases. Because of low proton fraction $\left(x_{p} \sim 0.05\right)$ in our models the dominant neutrino emission channel in the unpaired phases of hadrons is the modified Urca process. Additional smaller contribution from the modified bremsstrahlung process is also included. We use emissivities derived for the free pion-exchange model of strong interaction [62], but reduce them by a constant factor 5 to account for short-range repulsive component of the nuclear force [15, 16]. The effective mass corrections are included as well.

Below the critical temperatures $\left(T_{c}\right)$ for neutrons and protons the emissivities of these processes are suppressed, at asymptotically low temperatures exponentially. In general the emissivity of the modified Urca process in the superfluid phase can be expressed via its rate in the normal matter as follows

$$
\begin{aligned}
\epsilon_{\beta \text { mod }}\left(T \leq T_{c}\right) & =\epsilon_{\beta \text { mod }}\left(T>T_{c}\right) \\
& \times J_{\beta \text { mod }} \exp \left(-\frac{m \Delta_{n}(T)+k \Delta_{p}(T)}{T}\right),
\end{aligned}
$$

where $m$ and $k$ are integers and $J_{\beta \text { mod }}$ is a power-law function of $\Delta_{p} / T$ and $\Delta_{n} / T$, where $\Delta_{n, p}$ are the pairing gaps of protons and neutrons. The emissivities of the nucleonic superconducting phases were derived in Refs. [63, 64] for both modified and direct Urca as well as in Ref. [65, 66] for the direct Urca process using different methods. Below, for sake of simplicity, we assume $m=k=1$ as well as $J_{\beta \bmod }=1$. This approximation captures approximately the low-temperature asymptotic 
reduction of the emissivity, but is a rather crude approximation at $T \leq T_{c}$. More detailed expressions are not needed for the description of cooling of hybrid stars, which is dominated by the processes involving quarks. The neutrino emission processes from Cooper PBF start to contribute below respective $T_{c}$ [13, 14]. The neutral vector current processes are strongly suppressed by multiloop contribution to the response function of $S$-wave paired condensate [17, 18, 19, 20]. The axial-vector emission can be taken at one-loop level to a good accuracy. For $P$-wave paired neutrons the rates are unaffected by the multiloop processes and we use the results of Refs. [21, 22]. Given the zero-temperature values of the nucleonic gaps $\Delta(0)$, their finite-temperature values are well approximated by the asymptotic expressions given in Ref. [67] (as well as fits to tables in that work [68])

$$
\frac{\Delta(T)}{\Delta(0)}=\left\{\begin{array}{cc}
1-\sqrt{2 \gamma \tau} e^{-\pi /(\gamma \tau)} & 0 \leq \tau \leq 0.5, \\
\sqrt{3.016(1-\tau)-2.4(1-\tau)^{2}} & 0.5<\tau \leq 1,
\end{array}\right.
$$

where $\Delta(0)$ is the pairing gap in the zero-temperature limit, $\tau=T / T_{c}$ is the temperature in units of the critical temperature and $\gamma=1.781$. The formulae (8) reproduce the solution of the BCS gap equation with zero-range interaction within a percent accuracy. Crustal neutrino emission contributes to the cooling of the star in the final stages of neutrino cooling era via the electron neutrino bremsstrahlung emission on nuclei in the crusts [70]. Ions in the crust may form a fluid or a solid. The abundances of impurities, if high, could lead to additional the neutrino emission. In the fluid or impurity dominated crust the emissivity scales as $T^{6}$ and we adopt this option for temperatures relevant for that particular cooling phase $\left(T \leq 10^{9} \mathrm{~K}\right)$. If crystalline lattice is formed at the temperatures of interest, then the emissivities would scale approximately as $T^{7}$ (classical crystal) or as $T^{8}$ (quantum crystal) and the bremsstrahlung emissivity is parametrically suppressed [71].

\subsection{Specific heat}

The temperature dependence of the specific heat of normal Fermi-liquids (both relativistic and non-relativistic) is given by the linear law $c_{V}=a T$, where the coefficient depends on the abundance of given species (which is the same as the Fermi momentum). We apply this formula to the leptonic component of the star and unpaired hadrons and quarks.

As well known, in ordinary BCS superconductors the specific heat as a function of temperature experiences a jump at the corresponding critical temperature and then decays, at low temperatures exponentially. For $S$-wave superconductors one can apply the a asymptotic expressions given in Ref. [67] (as well as fits to tables in that work [68]) to model the ratio of the specific heats in the superconducting $c_{S}$ and normal $c_{N}$ phases

$$
\frac{c_{S}(T)}{c_{N}\left(T_{c}\right)}=\left\{\begin{array}{cc}
(12 \pi / \gamma)(2 \gamma \tau)^{-3 / 2} e^{-\pi /(\gamma \tau)} & 0 \leq \tau \leq 0.3, \\
-0.24422+0.255292 \tau & 0.3<\tau \leq 1, \\
+2.43077 \tau^{2} & 0.3 \leq 1
\end{array}\right.
$$

where $T_{c}$ is the critical temperature of phase transition. Equation (9) was applied uniformly to neutron, proton, and bluequark condensates. See Ref. [69] for possible effects of $P$-wave superfluidity of neutrons in the core on their specific heat.
To find the specific heat of the red-green condensate we need an expression for the critical temperature as a function of the mismatch $\delta \mu$. We use the following formula

$$
T_{c}(\zeta) \simeq T_{c 0} \sqrt{1-\frac{4 \mu}{3 \Delta_{0}} \delta(\zeta)}
$$

where $\mu=\left(\mu_{d}+\mu_{u}\right) / 2, \Delta_{0}=\Delta(\zeta=0), T_{c 0}=T_{c}(\zeta=0)$, and $\delta(\zeta)=\left(n_{d}-n_{u}\right) /\left(n_{d}+n_{u}\right)$.

As in the case of emissivities, the availability of gapless fermions in the case $\zeta \leq 1$ changes the BCS behaviour of the specific heat as well. To model this regime we apply, in analogy to emissivities, the following formula

$$
\frac{c_{S}^{r g}\left(\zeta ; T \leq T_{c}\right)}{c_{N}^{r g}}=f(\zeta)
$$

where $c_{N}^{r g}$ is the specific heat of red-green unpaired quarks, taken as that for noninteracting quarks and $c_{S}^{r g}$ is the specific heat of pair-correlated quarks.

\section{Results of numerical simulations}

\subsection{General considerations}

The thermal evolution code employed in our study uses the isothermal core approximation which is valid for timescales that are larger that those which are required to dissolve temperature gradients by thermal conduction. In the hadronic core the thermal conductivity is dominated by the electron transport if baryons are superfluid [72] and baryons if these are nonsuperfluid [73]. The characteristic times-scales for thermal relaxation are of the order of $t \sim 100 \mathrm{y}$. In the 2SC phase likewise the transport is dominated by electrons and blue-quarks [74]. the early stage of thermal evolution of 2SC phase and the timescale of its thermal relaxation remains to be studied; here we assue that the entire (quark plus hadronic) core is thermally relaxed at the start of simulation. Note that for reasonable initial temperatures the cooling tracks exit the non-isothermal phase and settle at a temperature predicted by the balance of the dominant neutrino emission mechanism and the specific heat of the core independent of the earlier evolution phase.

The isothermal core is defined by the transition density $\rho_{\text {tr }}=$ $10^{10} \mathrm{~g} \mathrm{~cm}^{-3}$. At lower densities the envelope maintains temperature gradients throughout the complete evolution. The physics of thermal transport can be encoded in a relation between the interior and surface temperatures. The models of envelopes predict the scaling $T_{s}^{4}=g_{s} h(T)$, where $T_{s}$ is the surface temperature $T$ is the the isothermal core temperature, $g_{s}$ is the surface gravity, and $h$ is a function which depends on $T$, the opacity of crustal material, and its EoS. This can be written as [75, 76]

$$
T_{s 6}=\left(\alpha T_{9}\right)^{\beta} g_{s}^{1 / 4}
$$

where $T_{9}$ the isothermal core temperature in units of $10^{9} \mathrm{~K}$, $T_{s 6}$ is the surface temperature in units of $10^{6} \mathrm{~K}, \alpha$ and $\beta$ are constants that depend on the composition of the star's atmosphere, $g_{s}$ is the surface gravity in units of $10^{14} \mathrm{~cm} \mathrm{~s}^{-2}$. We 


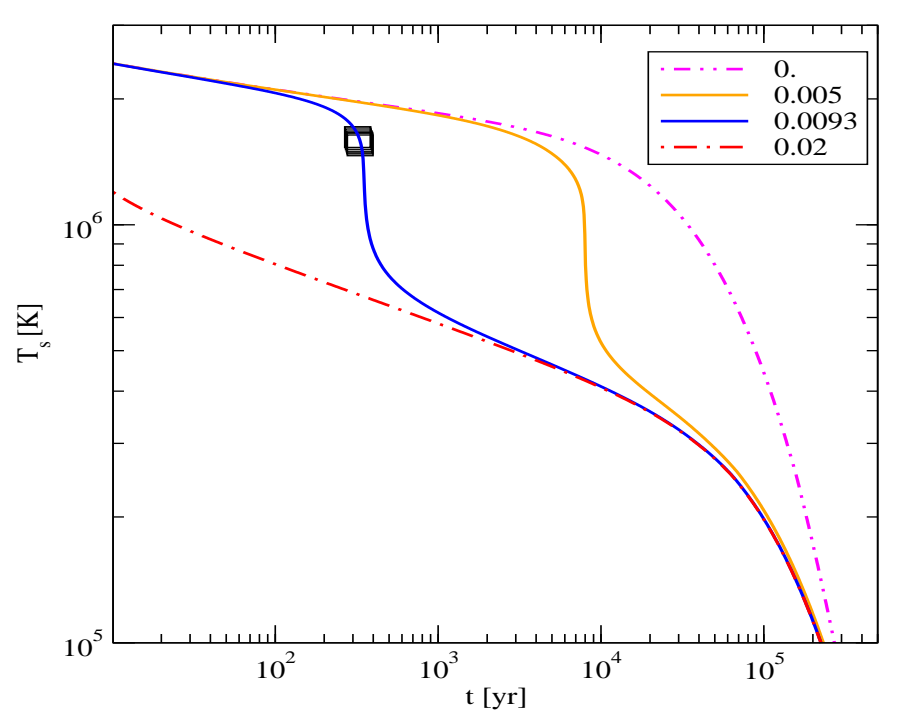

Fig. 3. Dependence of the surface temperature of a CSs given in $\mathrm{K}$ on time in year. The lines correspond to different phase transition temperature $T^{*}$ for fixed values of the width $w=0.2$ and the blue quark pairing gap $\Delta_{b}=0.15$ (both in $\mathrm{MeV}$ ). The labels correspond to values of $T^{*}$ in $\mathrm{MeV}$. The Cas A data is shown by squares.

use $\beta=0.55$, which lies between the values for the purelyiron $(\beta=0.5)$ and the fully accreted $(\beta=0.61)$ envelope and $\alpha=18.1$, which is appropriate for an accreted envelope [76]. Our fit results are sensitive to the $\beta$ parameter, but not to the $\alpha$ parameter. With these ingredients at hand we have numerically integrated the thermal evolution equation as described in Ref. [4].

The numerical simulations were carried out for all models listed in Table 1, but we concentrate below mostly on the representative model with $M / M_{\odot}=1.93$. Three parameters were varied: (a) the transition temperature $T^{*}$ from the $2 \mathrm{SC}$ to the crystalline phase; (b) the blue quark gap $\Delta_{b}$; and (c) the width of the transition $w$. The remaining parameters which model the temperature (time) dependent part of the $\zeta$ function were held fixed at values $\zeta_{i}=1.1$ and $\Delta \zeta=0.2$. The constant zerotemperature value of the red-green gap is fixed at the value $\Delta_{r g}=60 \mathrm{MeV}$. Figure 3 shows the dependence of the (redshifted) surface temperature on time for $M / M_{\odot}=1.93$ stellar model and for different values of the $T^{*}$ (as indicated in the figure) with $w$ and $\Delta_{b}$ fixed. In the limit $T^{*} \rightarrow 0$ the quark core does not influence the cooling because the neutrino emission from the red-green and blue condensates is suppressed for $T \ll \Delta_{r g}$ and $T \ll \Delta_{b}$. For high values of $T^{*}(0.2 \mathrm{MeV}$ in the figure) the transition to the FF phase occurs early in the evolution of the star, therefore enhanced neutrino emission cools the star rapidly below the value observed for the CS in Cas A. It is further seen that by tuning the phase-transition temperature to the value $T^{*}=0.009385 \mathrm{MeV}$ the "drop" in the temperature of the model can be adjust to the observed temperature and age of the CS in Cas A. Thus, if the current interpretation is correct, then the CS is a massive compact star (so it features a quark core) and it undergoes currently a phase transition from the 2SC to the FF phase (or some other gapless phase). Note that the deduced phase transition temperature is

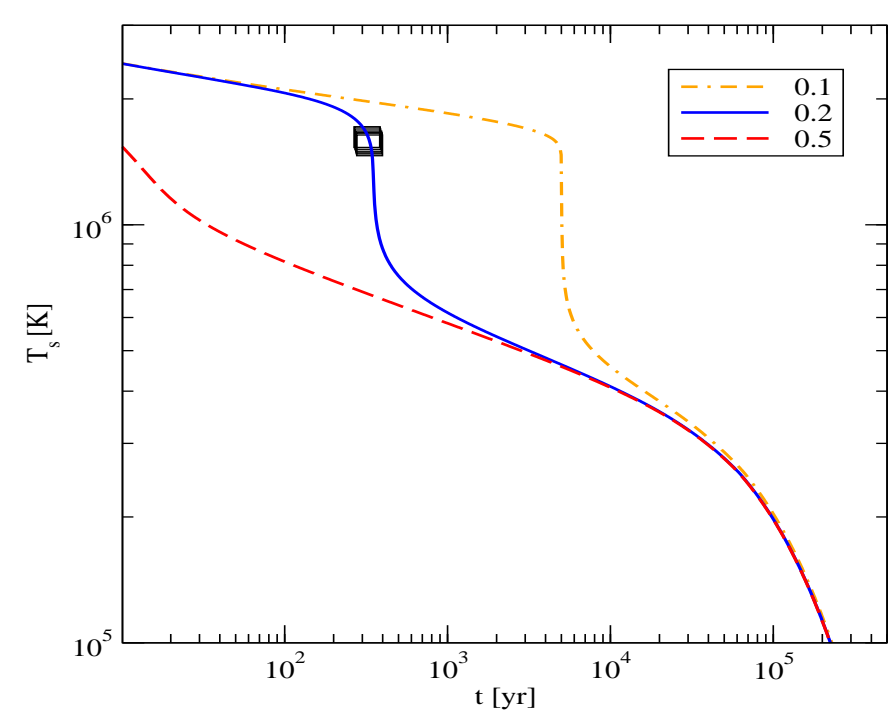

Fig. 4. Same as in fig. 3 but for different values of the width $w$ for fixed $T^{*}=0.009385$ and $\Delta_{b}=0.15 \mathrm{MeV}$. The labels correspond to values of $w$ in $\mathrm{MeV}$.

of the order of $0.01 \mathrm{MeV}$ and is small compared to the scale set by the $\Delta_{r g}$. This could be the feature of the equilibrium phase diagram of the superconducting quark matter or alternatively may reflect the non-equilibrium aspect of the phase transition, i. e., long-lived metastable $2 \mathrm{SC}$ phase (in parallel to super-heating or super-cooling of superconducting states, know from the physics of ordinary superconductors). We conclude that with a proper choice of the value of the transition temperature we can account for the observed "drop" in the temperature.

Next we fix the parameters $T^{*}$ and $\Delta_{b}$ and vary the width of the transition $w$, which controls the "smoothness" of the transition (fig. (4). For small values of $w$ ( 0.1 in the figure) the transient is steeper, as expected, but is also delayed (i. e. the drop is shifted away from the Cas A location to later times). Inversely, large $w$ make the transient much smoother and also the start of the phase transition is shifted to the left, i. e., to earlier times. Thus, small (by a factor of 2) variation of the parameters $w$ and $T^{*}$ can cause substantial shifts in the cooling curves, i. e., the overall fits to the Cas A data are sensitive to the values of these parameters. Finally we fix the parameters $T^{*}$ and $w$ and vary the value of the gap of blue quarks $\Delta_{b}$ (fig. 5). Increasing the magnitude of $\Delta_{b}$ shits the transient to later times, but also the pre-transient temperatures increase because of the exponential suppression factor in eq. (6) is less effective. Inversely, decreasing the value of $\Delta_{b}$ shifts the transient to earlier times and decreases the transient temperature. This behaviour is consistent with the fact that the cooling for heavy models is dominated by the red-green and blue quark components. In the 2SC phase the cooling is controlled by the Urca process on blue quarks, which is suppressed for late times and low temperatures, see Eq. (6). Unless the value of $\Delta_{b}$ is too small the blue quarks dominate throughout the neutrino cooling era. At later stages $t \sim 10^{5}$ y the photon emission becomes the main cooling mechanism (this is indicated by the change of the slope of the cooling curve). During and after the transient, the gapless 


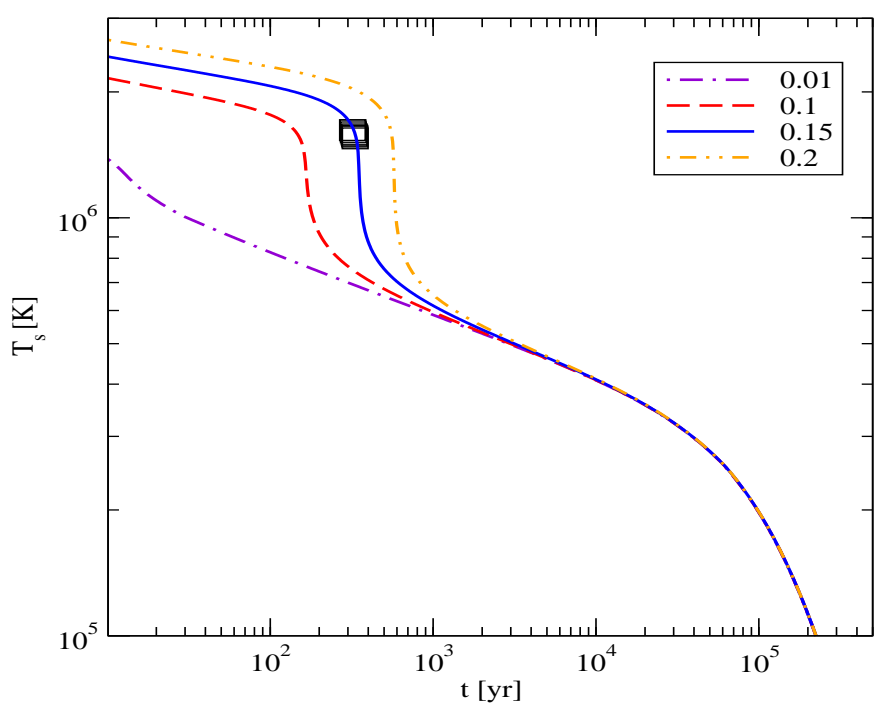

Fig. 5. Same as in fig. 3 but for different values of the blue quark gap $\Delta_{b}$ for fixed $T^{*}=0.009385$ and $w=0.2 \mathrm{MeV}$. The labels correspond to values of $\Delta_{b}$ in $\mathrm{MeV}$.

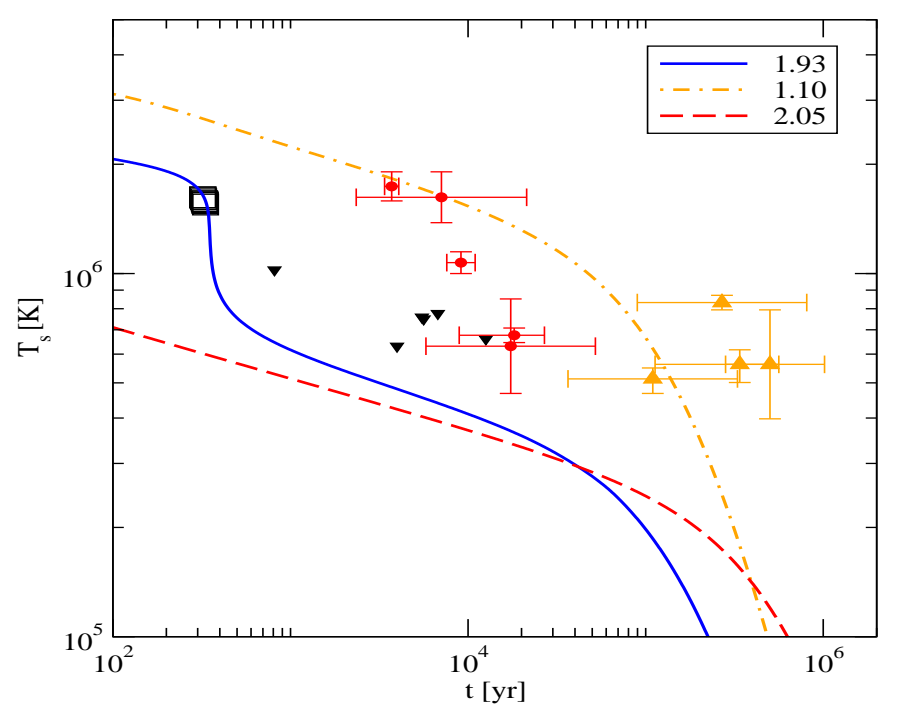

Fig. 6. Same as in fig. 3 for fixed $T^{*}=0.009385$ but for different masses of CS. The labels correspond to masses of the stars in units $M / M_{\odot}$. The references in the data are given in fig. 3 of Ref. [4].

phase emits neutrinos on red-green quarks; their luminosity is larger by factor two - the number of involved flavors - compared to blue quark luminosity. In this case again the transition is to the photon cooling at $t \sim 10^{5} \mathrm{y}$. An important conclusion is that for massive CS the neutrino cooling is completely dominated by quarks and the details of the modeling of hadronic neutrino cooling are irrelevant. This implies, obviously, that the quality of the fits to the entire population of thermally emitting pulsars is not correlated to our fits to the Cas A data, as this population needs to include purely hadronic neutron stars with $M \leq 1.8 M_{\odot}$, which will cool much slowly.

We show in fig. 6 the variation of cooling curves with the mass of the CS holding the "best" values of the parameters fit-

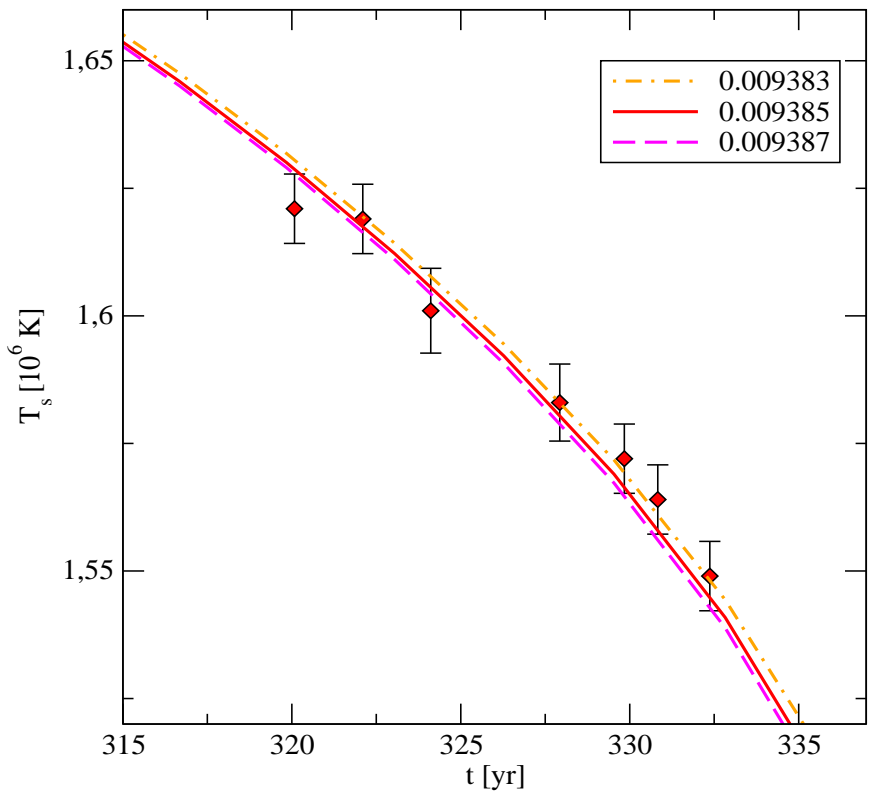

Fig. 7. Dependence of the surface in units of $10^{6} \mathrm{~K}$ temperature on time for several temperatures of the phase transition $T^{*}$ at fixed $w=$ 0.2 and $\Delta_{b}=0.15$ (both in $\mathrm{MeV}$ ). The dots with error bars show the Cas A data taken from [5].

ting Cas A fixed. It is seen that there is a substantial variation in the cooling speed of CSs with the mass; the heavy stars are cooling much faster than the light stars, for the neutrino emissivity of the hadronic phases is less effective that those of the quark phases. Note also the inversion of the temperatures in the photon cooling era: here the heavier $M / M_{\odot}=2.05$ star is hotter than the lighter $M / M_{\odot}=1.93$ star. The band lying between the cooling curves of extrem mass object, i.e., $1.1 \leq M / M_{\odot} \leq 2.05$ in the $T-t$ plane can be covered by changing the central density (and therefore the mass) of the model and the experimental data can naturally be accommodated. (The few outliers, which are hot at late stages of evolution $t \sim 10^{6} \mathrm{y}$, may reflect different surface composition, than assumed in our simulations, e.g., composition containing hydrogen). Thus, we conclude that the spread in the temperatures of thermally emitting neutron stars can be attributed to the different masses of these objects: the lighter ones not containing quark matter cool more slowly than the heavier stars having quark cores. Of course, variations in other factors, such as the strength of the magnetic field, the surface composition, etc can also contribute to the dispersion.

\subsection{Fits to the Cas A data}

The Cas A data spanning the decade 2003-13 have been used for the fits. It is assumed that the star is already in the transient stage, as there is no evidence of the entry into this phase from a slower cooling stage, as well as no sign of the exit from the fast cooling mode. The parameters were adjusted to obtain the location of the Cas A data in the $T-t$ diagram as well as the slope of the transient. The best fit is obtained with the parameter values $T^{*}=0.009385, w=0.2$ and $\Delta_{b}=0.15$ (all quantities in $\mathrm{MeV}$ ). The sensitivity of fits was tested against variation of 


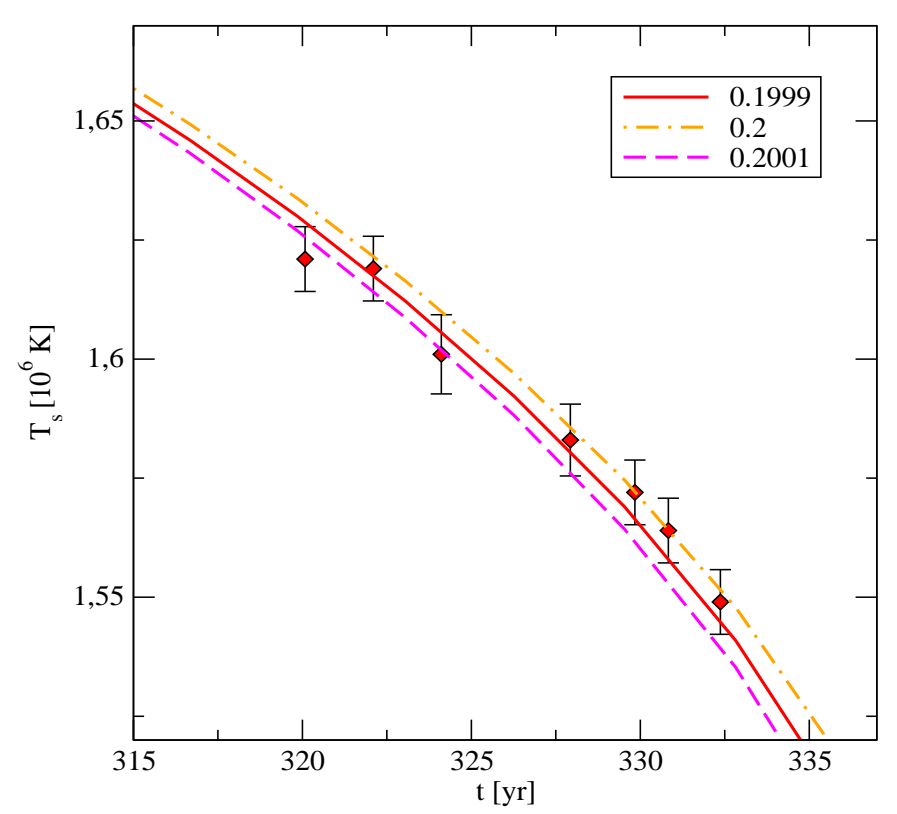

Fig. 8. Same as in 7 but for a range of values of $w$ indicated in the plot and for fixed values of $T^{*}=0.009385$ and $\Delta_{b}=0.15$. All parameter values are in $\mathrm{MeV}$.

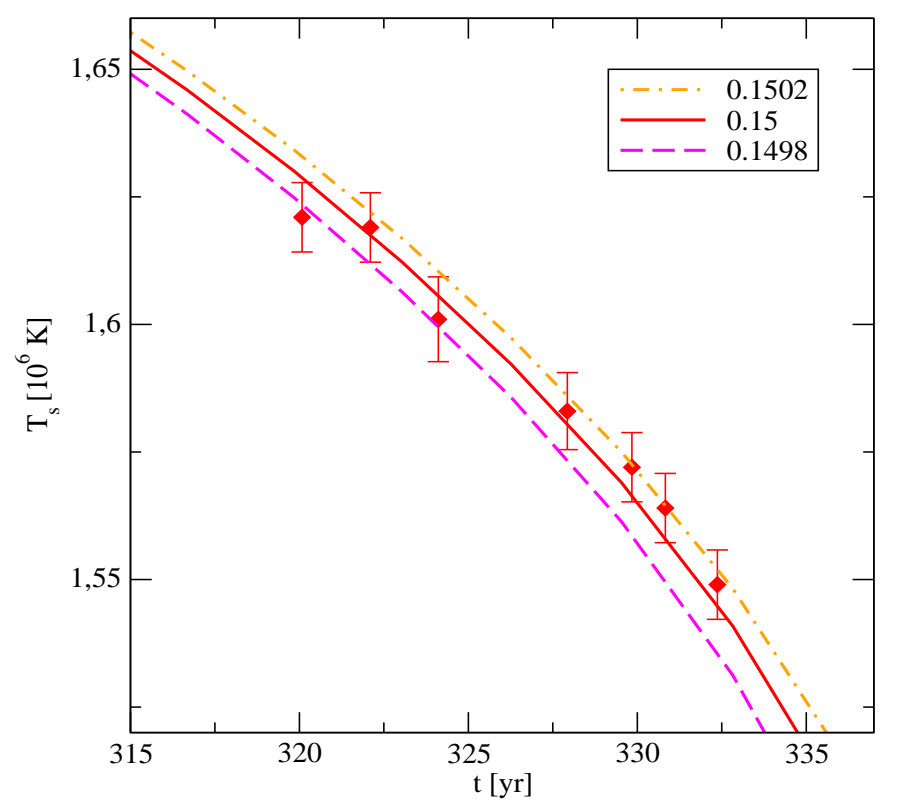

Fig. 9. Same as in 7 but for a range of values of $\Delta_{b}$ indicated in the plot and for fixed values of $T^{*}=0.009385$ and $w=0.2$. All parameter values are in $\mathrm{MeV}$.

one of them at fixed values of the other two. Figure 7 shows the effect of variations of $T^{*}$ on the transient behaviour. We quantify the sensitivity of the fits by defining the relative deviation as $\delta T^{*} / T^{*}$, where $\delta T^{*}$ is the range of values of $T^{*}$ compatible with the data; we find that $\delta T^{*} / T^{*} \simeq 10^{-3}$. Figure 8 shows the effect of variation of the width $w$ at fixed values of other two parameters. In this case we find that $\delta w / w=1.5 \times 10^{-3}$, i.e., the relative deviation is in the same range as for the transition temperature $T^{*}$. Figure 9 shows the variation in the value of the blue quark gap $\Delta_{b}$; in this case we find that $\delta \Delta_{b} / \Delta_{b} \simeq 3.3 \times 10^{-3}$.

Thus we conclude that the fitted values are quite sensitive to the precise values of parameters and small deviations at the level of $10^{-3}$ can spoil the fit to the Cas A data. Extrapolating from the present fits one would predict that the CS in Cas A would continue the fast cooling up to temperatures somewhat below $10^{6} \mathrm{~K}$. Clearly, an observation of an exit from the transient would provide further strong constraints on any model of the behaviour of the CS in Cas A and current fits should by updated to account for that effect.

\section{Summary and conclusions}

The observation of two-solar mass pulsars in binary systems is a strong evidence that the EoS of dense matter must be stiff and that the densities in the centers of compact stars can be large enough (several times the saturation density) so that the threshold density for transition to quark matter can be reached. This motivates the studies of the dense phases of quark matter under the conditions expected in compact stars (charge and color neutrality, near-equilibrium with respect to weak processes). We have concentrated here on CSs that have sharp interface between the hadronic and quark matter phases, in which case the massive members of the sequences $\left(M>1.85 M_{\odot}\right)$ contain progressively larger amount of quark matter whereas the lighter members are purely hadronic. We have evolved these models in time and followed the changes in the core and surface temperature under assumption that the core is isothermal, which is a valid approximation, except for the first $100 \mathrm{y}$ following the birth of the star.

The generic phase diagram of imbalanced fermionic systems, which also includes the flavor asymmetric pairing among quarks, implies that at high temperatures the pairing is in the BCS type phase, i.e., both Fermi surfaces of quarks are gapped despite of the mismatch between the Fermi surfaces of $u$ and $d$ flavors. At lower temperatures a transition from the BCS to a generic gapless phase must take place. This gapless phase will have some type of spatial modulation and we considered the FF type simple realization of such phase. The neutrino emissivity of such phase must be much larger than the emissivity of the 2SC phase because of the existence of gapless excitations. We have modelled the emissivities of both phase in terms of a simple parameterization, which however takes into account the fact that as the temperature is lowered there is a phase transition from the $2 \mathrm{SC}$ to FF phase.

The rapid cooling of the CS in Cas A can be accounted for via the phase transition described above; we stress again that the phase transition takes place within the phase diagram of QCD and is the consequence of the ordering of various superconducting phases in the temperature, density, and isospin spaces. Such ordering was observed in numerous studies of other imbalance systems such as the hadronic superfluids or ultra-cold atomic condensates.

The Cas A data can be fitted by varying the phase transition temperature $T^{*}$ at fixed value of the remaining physical parameters - the width of the phase transition (or, equivalently, its duration) and the gap in the spectrum of BCS-paired blue quarks. It turns out that the relative accuracy of order $10^{-3}$ is 
required to fit the data, i. e., larger deviation will spoilt the fit. We also tested the sensitivity of the other two parameters to the variations when the remaining two parameters are fixed. The relative accuracy is again in the range $10^{-3}$. Assuming that the present model of cooling of CS in Cas A is correct, we immediately conclude that only the massive members of CS sequences that contain (color-superconducting) quark matter can undergo transients of this type. This is the main difference of our model to the hadronic alternatives which work also for low (or canonical $1.4 M_{\odot}$ ) stars. It is tempting to think that the various models of cooling of CS in Cas A can be distinguished through the measurements of the mass or other integral parameters of the CS in the future.

In addition to the Cas A behaviour we also studied the effect of variations of the mass of the CSs on their cooling behaviour. First, we find that larger (close to the maximum) mass stars will not show the same transient as the assumed model with $1.93 M_{\odot}$ mass; rather they will cool to lower temperatures much faster. As our code does not cover the very early evolution of CSs, we cannot resolve the transient that takes place very early in the evolution of a highly massive CS. Secondly, we find that low mass stars $M \sim 1.1 M_{\odot}$ are much hotter than their heavy counterparts, so that the band covered by the stars in the range $1.1 \leq M / M_{\odot} \leq 2$ can account for the measured surface temperatures of thermally emitting CS.

There are a number of issues that need further attention: (a) The appearance of strangeness in form of hyperons in the hadronic phase and strange quarks in the quark phases can substantially affect cooling. In particular strange quarks may even change the structure of the phase diagram of color superconducting matter at high density by inducing new phases and new channels for neutrino emission see, for example, Ref. [32]; (b) heating in the core and crust of the star can become important at later stages of evolution; it does not affect the cooling at the age of CS in Cas A (if the CS is not a magnetar); (c) the density dependence of pairing gaps of red-green and blue condensates can be a factor. The density of states at the Fermi surface increases as $1 / 3$ power of density, whereas the strong coupling constant decreases logarithmically. Therefore, one can envision a situation where the red-green condensate has $\zeta<1$ only in part of the core. This will reduce the effective volume of the quark phase contributing to the fast cooling; (d) the superconducting quark phases may gradually appear as a result of deceleration of the star, i.e., the CS can experience a rotation induced phase transition to a color superconducting phase [51]. This transition will occur in the densest region (center of the CS) in parallel with deconfinement of quarks.

\section{Acknowledgment}

This work ws supported by the Deutsche Forschungsgemeinschaft (Grant No. SE 1836/3-1) and by the NewCompStar COST Action MP1304.

\section{References}

1. N. Brambilla, S. Eidelman, P. Foka, S. Gardner, A. S. Kronfeld, M. G. Alford, R. Alkofer, M. Butenschoen,
T. D. Cohen, J. Erdmenger, L. Fabbietti, M. Faber, J. L. Goity, B. Ketzer, H. W. Lin, F. J. Llanes-Estrada, H. Meyer, P. Pakhlov, E. Pallante, M. I. Polikarpov, H. Sazdjian, A. Schmitt, W. M. Snow, A. Vairo, R. Vogt, A. Vuorinen, H. Wittig, P. Arnold, P. Christakoglou, P. Di Nezza, Z. Fodor, X. G. i Tormo, R. Höllwieser, A. Kalwait, D. Keane, E. Kiritsis, A. Mischke, R. Mizuk, G. Odyniec, K. Papadodimas, A. Pich, R. Pittau, J.-W. Qiu, G. Ricciardi, C. A. Salgado, K. Schwenzer, N. G. Stefanis, G. M. von Hippel, and V. I . Zakharov. QCD and strongly coupled gauge theories: challenges and perspectives. Eur. Phys. J. C, 74(12):2981, April 2014.

2. M. Buballa, V. Dexheimer, A. Drago, E. Fraga, P. Haensel, I. Mishustin, G. Pagliara, J. Schaffner-Bielich, S. Schramm, A. Sedrakian, and F. Weber. EMMI rapid reaction task force meeting on quark matter in compact stars. Journal of Physics G Nuclear Physics, 41(12):123001, December 2014.

3. A. Sedrakian. Rapid cooling of Cassiopeia A as a phase

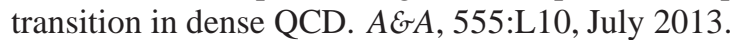

4. D. Hess and A. Sedrakian. Thermal evolution of massive compact objects with dense quark cores. Phys. Rev. D, 84(6):063015, September 2011.

5. K. G. Elshamouty, C. O. Heinke, G. R. Sivakoff, W. C. G. Ho, P. S. Shternin, D. G. Yakovlev, D. J. Patnaude, and L. David. Measuring the Cooling of the Neutron Star in Cassiopeia A with all Chandra X-Ray Observatory Detectors. ApJ, 777:22, November 2013.

6. B. Posselt, G. G. Pavlov, V. Suleimanov, and O. Kargaltsev. New Constraints on the Cooling of the Central Compact Object in Cas A. ApJ, 779:186, December 2013.

7. D. Page, M. Prakash, J. M. Lattimer, and A. W. Steiner. Rapid Cooling of the Neutron Star in Cassiopeia A Triggered by Neutron Superfluidity in Dense Matter. Physical Review Letters, 106(8):081101, February 2011.

8. P. S. Shternin, D. G. Yakovlev, C. O. Heinke, W. C. G. Ho, and D. J. Patnaude. Cooling neutron star in the Cassiopeia A supernova remnant: evidence for superfluidity in the core. MNRAS, 412:L108-L112, March 2011.

9. P. S. Shternin and D. G. Yakovlev. Self-similarity relations for cooling superfluid neutron stars. MNRAS, 446:3621-3630, February 2015.

10. W. C. G. Ho, K. G. Elshamouty, C. O. Heinke, and A. Y. Potekhin. Tests of the nuclear equation of state and superfluid and superconducting gaps using the Cassiopeia A neutron star. Phys. Rev. C, 91(1):015806, January 2015.

11. W. G. Newton, K. Murphy, J. Hooker, and B.-A. Li. The Cooling of the Cassiopeia A Neutron Star as a Probe of the Nuclear Symmetry Energy and Nuclear Pasta. ApJ, 779:L4, December 2013.

12. L. B. Leinson. Superfluid phases of triplet pairing and rapid cooling of the neutron star in Cassiopeia A. Phys. Lett. B, 741:87, 2015.

13. E. Flowers, M. Ruderman, and P. Sutherland. Neutrino pair emission from finite-temperature neutron superfluid and the cooling of young neutron stars. ApJ, 205:541544, April 1976.

14. A. V. Senatorov and D. N. Voskresenskii. Collective excitations in nucleonic matter and the problem of cooling of 
neutron stars. Physics Letters B, 184:119-124, Jan. 1987.

15. D. Blaschke, G. Röpke, H. Schulz, A. D. Sedrakian, and D. N. Voskresensky Nuclear in-medium effects and neutrino emissivity of neutron stars. MNRAS, 273:596-602, April 1995.

16. R. G. Timmermans, A. Y. Korchin, E. N. van Dalen, and A. E. Dieperink. Soft electroweak bremsstrahlung: Theorems and astrophysical relevance. Phys. Rev. C, 65:064007, June 2002.

17. L. B. Leinson and A. Pérez. Vector current conservation and neutrino emission from singlet-paired baryons in neutron stars. Physics Letters B, 638:114-118, July 2006.

18. A. Sedrakian, H. Müther, and P. Schuck. Vertex renormalization of weak interactions and Cooper-pair breaking in cooling compact stars. Phys. Rev. C, 76(5):055805, November 2007.

19. E. E. Kolomeitsev and D. N. Voskresensky. Neutrino emission due to Cooper-pair recombination in neutron stars reexamined. Phys. Rev. C, 77(6):065808, June 2008.

20. A. Sedrakian. Vertex renormalization of weak interactions in compact stars: Beyond leading order. Phys. Rev. C, 86(2):025803, August 2012.

21. D. G. Yakovlev, A. D. Kaminker, and K. P. Levenfish. Neutrino emission due to Cooper pairing of nucleons in cooling neutron stars. AEFA, 343:650-660, March 1999.

22. L. B. Leinson. Neutrino emissivity of ${ }^{3} \mathrm{P}_{2}-{ }^{3} \mathrm{~F}_{2}$ superfluid cores in neutron stars. Phys. Rev. C, 84(4):045501, October 2011.

23. D. Page, J. M. Lattimer, M. Prakash, and A. W. Steiner. Neutrino Emission from Cooper Pairs and Minimal Cooling of Neutron Stars. ApJ, 707:1131-1140, December 2009.

24. D. Blaschke, H. Grigorian, D. N. Voskresensky, and F. Weber. Cooling of the neutron star in Cassiopeia A. Phys. Rev. C, 85(2):022802, February 2012.

25. D. Blaschke, H. Grigorian, and D. N. Voskresensky. Nuclear medium cooling scenario in the light of new Cas A cooling data and the $2 M_{\odot}$ pulsar mass measurements. Phys. Rev. C, 88:065805, December 2013.

26. C. Schaab, D. Voskresensky, A. D. Sedrakian, F. Weber, and M. K. Weigel. Impact of medium effects on the cooling of non-superfluid and superfluid neutron stars. $A \mathcal{E} A$, 321:591-604, May 1997.

27. R. Negreiros, S. Schramm, and F. Weber. Impact of rotation-driven particle repopulation on the thermal evolution of pulsars. Physics Letters B, 718:1176-1180, January 2013.

28. T. Noda, M.-a. Hashimoto, N. Yasutake, T. Maruyama, T. Tatsumi, and M. Fujimoto. Cooling of Compact Stars with Color Superconducting Phase in Quark-hadron Mixed Phase. ApJ, 765:1, March 2013.

29. P. B. Demorest, T. Pennucci, S. M. Ransom, M. S. E. Roberts, and J. W. T. Hessels. A two-solar-mass neutron star measured using Shapiro delay. Nature, 467:10811083, October 2010.

30. J. Antoniadis, P. C. C. Freire, N. Wex, T. M. Tauris, R. S. Lynch, M. H. van Kerkwijk, M. Kramer, C. Bassa, V. S. Dhillon, T. Driebe, J. W. T. Hessels, V. M. Kaspi, V. I.
Kondratiev, N. Langer, T. R. Marsh, M. A. McLaughlin, T. T. Pennucci, S. M. Ransom, I. H. Stairs, J. van Leeuwen, J. P. W. Verbiest, and D. G. Whelan. A Massive Pulsar in a Compact Relativistic Binary. Science, 340:448, April 2013.

31. D. Page, M. Prakash, J. M. Lattimer, and A. W. Steiner. Prospects of Detecting Baryon and Quark Superfluidity from Cooling Neutron Stars. Physical Review Letters, 85:2048-2051, September 2000.

32. M. Alford, P. Jotwani, C. Kouvaris, J. Kundu, and K. Rajagopal. Astrophysical implications of gapless colorflavor locked quark matter: A hot water bottle for aging neutron stars. Phys. Rev. D, 71(11):114011, June 2005.

33. R. Anglani, G. Nardulli, M. Ruggieri, and M. Mannarelli. Neutrino emission from compact stars and inhomogeneous color superconductivity. Phys. Rev. D, 74(7):074005, October 2006.

34. H. Grigorian, D. Blaschke, and D. Voskresensky. Cooling of neutron stars with color superconducting quark cores. Phys. Rev. C, 71(4):045801, April 2005.

35. K. Rajagopal and F. Wilczek. The Condensed Matter Physics of QCD. arXiv: hep-ph/0011333, November 2000.

36. I. Shovkovy and M. Huang. Gapless two-flavor color superconductor. Physics Letters B, 564:205-211, July 2003.

37. H. Müther and A. Sedrakian. Breaking rotational symmetry in two-flavor color superconductors. Phys. Rev. D, 67(8):085024, April 2003.

38. M. Alford, J. A. Bowers, and K. Rajagopal. Crystalline color superconductivity. Phys. Rev. D, 63(7):074016, April 2001.

39. R. Anglani, R. Casalbuoni, M. Ciminale, N. Ippolito, R. Gatto, M. Mannarelli, and M. Ruggieri. Crystalline color superconductors. Reviews of Modern Physics, 86:509-561, April 2014.

40. A. Sedrakian and D. H. Rischke. Phase diagram of chiral quark matter: From weakly to strongly coupled FuldeFerrell phase. Phys. Rev. D, 80(7):074022, October 2009.

41. X.-G. Huang and A. Sedrakian. Phase diagram of chiral quark matter: Color and electrically neutral Fulde-Ferrell phase. Phys. Rev. D, 82(4):045029, August 2010.

42. M. Stein, A. Sedrakian, X.-G. Huang, and J. W. Clark. BCS-BEC crossovers and unconventional phases in dilute nuclear matter. Phys. Rev. C, 90(6):065804, December 2014.

43. M. Stein, A. Sedrakian, X.-G. Huang, J. W. Clark, and G. Röpke. Inhomogeneous condensates in dilute nuclear matter and BCS-BEC crossovers. Journal of Physics Conference Series, 496(1):012008, March 2014.

44. M. Stein, X.-G. Huang, A. Sedrakian, and J. W. Clark. Phase diagram of dilute nuclear matter: Unconventional pairing and the BCS-BEC crossover. Phys. Rev. C, 86(6):062801, December 2012.

45. M. G. Alford, J. A. Bowers, J. M. Cheyne, and G. A. Cowan. Single color and single flavor color superconductivity. Phys. Rev. D, 67(5):054018, March 2003.

46. A. Schmitt. Ground state in a spin-one color superconductor. Phys. Rev. D, 71(5):054016, March 2005. 
47. D. N. Aguilera, D. Blaschke, M. Buballa, and V. L. Yudichev. Color-spin locking phase in two-flavor quark matter for compact star phenomenology. Phys. Rev. D, 72(3):034008, August 2005.

48. N. D. Ippolito, M. Ruggieri, D. H. Rischke, A. Sedrakian, and F. Weber. Equilibrium sequences of nonrotating and rapidly rotating crystalline color-superconducting hybrid stars. Phys. Rev. D, 77(2):023004, January 2008.

49. B. Knippel and A. Sedrakian. Gravitational radiation from crystalline color-superconducting hybrid stars. Phys. Rev. D, 79(8):083007, April 2009.

50. L. Bonanno and A. Sedrakian. Composition and stability of hybrid stars with hyperons and quark colorsuperconductivity. AEFA, 539:A16, March 2012.

51. N. S. Ayvazyan, G. Colucci, D. H. Rischke, and A. Sedrakian. Rotating hybrid compact stars. AEFA, 559:A118, November 2013.

52. W. M. Spinella, F. Weber, G. A. Contrera, and M. G. Orsaria. Neutrino emissivity in the quark-hadron mixed phase of neutron stars. arXiv:1507.06067, July 2015.

53. G. Colucci and A. Sedrakian. Equation of state of hypernuclear matter: Impact of hyperon-scalar-meson couplings. Phys. Rev. C, 87(5):055806, May 2013.

54. G. Colucci and A. Sedrakian. Equation of state of hypernuclear matter: tuning hyperon-scalar-meson couplings. Journal of Physics Conference Series, 496(1):012003, March 2014.

55. E. N. E. van Dalen, G. Colucci, and A. Sedrakian. Constraining hypernuclear density functional with $\Lambda^{-}$ hypernuclei and compact stars. Physics Letters $B$, 734:383-387, June 2014.

56. K. A. Maslov, E. E. Kolomeitsev, and D. N. Voskresensky. Solution of the hyperon puzzle within a relativistic mean-field model. Physics Letters B, 748:369-375, September 2015.

57. M. Fortin, J. L. Zdunik, P. Haensel, and M. Bejger. Neutron stars with hyperon cores: stellar radii and equation

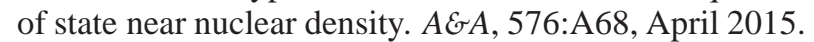

58. M. Oertel, C. Providência, F. Gulminelli, and A. R. Raduta. Hyperons in neutron star matter within relativistic mean-field models. Journal of Physics G Nuclear Physics, 42(7):075202, July 2015.

59. P. F. Bedaque and A. W. Steiner. Hypernuclei and the hyperon problem in neutron stars. Phys. Rev. C, 92(2):025803, August 2015.

60. N. Iwamoto. Quark beta decay and the cooling of neutron stars. Physical Review Letters, 44:1637-1640, June 1980.

61. P. Jaikumar, C. D. Roberts, and A. Sedrakian. Direct Urca neutrino rate in color superconducting quark matter. Phys. Rev. C, 73(4):042801, April 2006.

62. B. L. Friman and O. V. Maxwell. Neutrino emissivities of neutron stars. ApJ, 232:541-557, September 1979.

63. M. E. Gusakov. Neutrino Emission from Superfluid Neutron-Star Cores: Various Types of Neutron Pairing. AEA A, 389:702-715, July 2002.

64. D. G. Yakovlev, A. D. Kaminker, O. Y. Gnedin, P. Haensel. Neutrino emission from neutron stars. Physics Reports, 354:1-155, November 2001.
65. A. Sedrakian. Direct Urca neutrino radiation from superfluid baryonic matter. Phys. Lett. B, 607:27-34, November 2005 .

66. A. Sedrakian. The physics of dense hadronic matter and compact stars. Prog. Part. Nucl. Phys, 58:168-246, November 2007. 58:168-246,

67. B. Mühlschlegel. Die thermodynamischen Funktionen des Supraleiters. Zeitschrift fur Physik, 155:313-327, June 1959.

68. Daniel Hess. Cooling of hybrid compact star. Bachelor Thesis, Goethe-University, Frankfurt am Main, 2011.

69. K. P. Levenfish and D. G. Yakovlev. Specific heat of neutron star cores with superfluid nucleons. Astronomy Reports , 38:247-251, Mar 1994.

70. G. G. Festa and M. A. Ruderman. Neutrino-Pair Bremsstrahlung from a Degenerate Electron Gas. Physical Review, 180:1227-1231, April 1969.

71. A. D. Kaminker, C. J. Pethick, A. Y. Potekhin, V. Thorsson, and D. G. Yakovlev. Neutrino-pair bremsstrahlung by electrons in neutron star crusts. AE्FA, 343:1009-1024, March 1999.

72. E. Flowers and N. Itoh. Transport properties of dense matter. II. ApJ, 230:847-858, June 1979.

73. P. S. Shternin and D. G. Yakovlev. Electron-muon heat conduction in neutron star cores via the exchange of transverse plasmons. Phys. Rev. D, 75:103004, May 2007.

74. M. G. Alford, H. Nishimura, and A. Sedrakian. Transport coefficients of two-flavor superconducting quark matter. Phys. Rev. C, 90(5):055205, November 2014.

75. E. H. Gudmundsson, C. J. Pethick, and R. I. Epstein. Structure of neutron star envelopes. ApJ, 272:286-300, September 1983.

76. A. Y. Potekhin, G. Chabrier, and D. G. Yakovlev. Internal temperatures and cooling of neutron stars with accreted envelopes. AEFA, 323:415-428, July 1997. 